\title{
A NOTE ON AN INEQUALITY SIMILAR TO OPIAL'S INEQUALITY
}

\author{
B. G. PACHAPTTE
}

\begin{abstract}
A.bstract. In the present note we establish a new inequality similar to Opial's inequality by using a fairly elementary analysis.
\end{abstract}

\section{Introduction}

In a celebrated paper [4] Z. Opial proved the following inequality.

If $f \in C^{1}[0, h]$ satisfies $f(0)=f(h)=0$ and $f^{\prime}(x)>0$ for $x \in[0, h]$, then

$$
\int_{0}^{h}\left|f(x) f^{\prime}(x)\right| d x \leq \frac{h}{4} \int_{0}^{h}\left|f^{\prime}(x)\right|^{2} d x
$$

where the constant factor $\frac{h}{4}$ is best possible.

The Opial's inequality has evoked considerable interest since from its appearence and many papers dealing with new proofs, extensions, generalizations, variants and discrete analogues have appeared in the literature, see [1, 3, 5-8] and the references given therein. The aim of this note is to establish a new inequality similar to Opial's inequality (1) involving linear differential operator $L y$, its related initial value problem solution $y$, the associated Wronskin function $W$ and the initial conditions point $x_{0} \in R$. The analysis used in the proof is elementary and our result provides new estimate on this type of inequalities.

\section{Main Results}

Let $I$ be a closed interval of $R$, the set of real numbers. Let $a_{1}, \ldots, a_{n}, b$ be continuous functions on an interval $I$ and let $L=D^{n}+a_{1}(x) D^{n-1}+\cdots+a_{n}(x)$ be a fixed linear differential operator on $C^{n}(I)$. Let $\phi_{1}(x), \ldots, \phi_{n}(x)$ be a basis for the solutions of $L y=0$. Let $x_{0}$ be a fixed point in $I$, then it is well known (see, [2, pp.122-123]) that the unique solution to the initial value problem

$$
L y=b(x), \quad y^{(i)}\left(x_{0}\right)=0, \quad i=0,1,2, \ldots, n-1,
$$

Received July, 1998.

1991 Mathematics Subject Classification. 26D10, 26D15.

Key words and phrases. Opial's inequality, Wronskin function, differential operator, Hölder's inequality. 
is given by

$$
y(x)=\int_{x_{0}}^{x} \sum_{k=1}^{n} \phi_{k}(x) \sigma_{k}(t) b(t) d t
$$

where

$$
\sigma_{k}(t)=\frac{W_{k}\left(\phi_{1}, \ldots, \phi_{n}\right)(t)}{W\left(\phi_{1}, \ldots, \phi_{n}\right)(t)}
$$

in which $W\left(\phi_{1}, \ldots, \phi_{n}\right)$ is the Wronskian of the basis $\phi_{1}, \ldots, \phi_{n}$, and $W_{k}$ is the determinant obtained from $W\left(\phi_{1}, \ldots, \phi_{n}\right)$ by replacing the $k$-th column $\left(\phi_{k}, \phi_{k}^{\prime}, \ldots, \phi_{k}^{(n-1)}\right)$ by $(0,0, \ldots, 0,1)$.

Our main result is given in the following theorem.

Theorem 1. Let $x \geq x_{0}, x_{0}, x \in I$ and $p \geq 1, q>0$ be real constants. Let $U(x)$, $V(x)$ be real-valued positive continuous functions defined on $I$. Let $\phi_{1}, \ldots, \phi_{n}$ be a basis for the solutions of $L y=0$ and $y(x)$ be a unique solution of (2) on I. Then

$$
\int_{x_{0}}^{x} U(t)|y(t)|^{p}|(L y)(t)|^{q} d t \leq C A(x) \int_{x_{0}}^{x} V(t)|(L y)(t)|^{p+q} d t
$$

where $C=(q /(p+q))^{q /(p+q)}$ and

$$
\begin{aligned}
A(x)= & {\left[\int _ { x _ { 0 } } ^ { x } U ^ { ( p + q ) / p } ( t ) V ^ { - q / p } ( t ) \left[\int_{x_{0}}^{t} V^{-1 /(p+q-1)}(s)\right.\right.} \\
& \left.\left.\times\left(\sum_{k=1}^{n}\left|\phi_{k}(t) \sigma_{k}(s)\right|\right)^{(p+q) /(p+q-1)} d s\right]^{p+q-1} d t\right]^{p /(p+q)},
\end{aligned}
$$

for $x \geq x_{0}, x_{0}, x \in I$.

Proof. From the hypotheses, for $x_{0} \leq t \leq x$, we have

$$
y(t)=\int_{x_{0}}^{t} \sum_{k=1}^{n} \phi_{k}(t) \sigma_{k}(s)(L y)(s) d s .
$$

From (7) and using Hölder's inequality with indices $p+q$ and $(p+q) /(p+q-1)$ we obtain

$$
\begin{aligned}
|y(t)|^{p} \leq & {\left[\int_{x_{0}}^{t} \sum_{k=1}^{n}\left|\phi_{k}(t) \sigma_{k}(s)\right||(L y)(s)| d s\right]^{p} } \\
= & {\left[\int_{x_{0}}^{t} V^{-1 /(p+q)}(s) \sum_{k=1}^{n}\left|\phi_{k}(t) \sigma_{k}(s)\right| V^{1 /(p+q)}(s)|(L y)(s)| d s\right]^{p} } \\
\leq & {\left[\left(\int_{x_{0}}^{t} V^{-1 /(p+q-1)}(s)\left(\sum_{k=1}^{n}\left|\phi_{k}(t) \sigma_{k}(s)\right|\right)^{(p+q) /(p+q-1)} d s\right)^{(p+q-1) /(p+q)}\right.} \\
& \left.\times\left(\int_{x_{0}}^{t} V(s)|(L y)(s)|^{p+q} d s\right)^{1 /(p+q)}\right]^{p} .
\end{aligned}
$$


Multiplying both sides of (8) by $U(t)|(L y)(t)|^{q}$, integrating the resulting inequality over $\left[x_{0}, x\right]$ and using Hölder's inequality with indices $(p+q) / p$ and $(p+q) / q$, we obtain

$$
\begin{aligned}
& \int_{x_{0}}^{x} U(t)|y(t)|^{p}|(L y)(t)|^{q} d t \\
\leq & \int_{x_{0}}^{x}\left\{U ( t ) V ^ { - q / ( p + q ) } ( t ) \left[\int_{x_{0}}^{t} V^{-1 /(p+q-1)}(s)\right.\right. \\
& \left.\left.\times\left(\sum_{k=1}^{n}\left|\phi_{k}(t) \sigma_{k}(s)\right|\right)^{(p+q) /(p+q-1)} d s\right]^{p(p+q-1) /(p+q)}\right\} \\
& \times\left\{\left[\int_{x_{0}}^{t} V(s)|(L y)(s)|^{p+q} d s\right]^{p /(p+q)} V^{q /(p+q)}(t)|(L y)(t)|^{q}\right\} d t \\
\leq & {\left[\int _ { x _ { 0 } } ^ { x } U ^ { ( p + q ) / p } ( t ) V ^ { - q / p } ( t ) \left[\int_{x_{0}}^{t} V^{-1 /(p+q-1)}(s)\right.\right.} \\
& \left.\left.\times\left(\sum_{k=1}^{n}\left|\phi_{k}(t) \sigma_{k}(s)\right|\right)^{(p+q) /(p+q-1)} d s\right]^{p+q-1} d t\right]^{p /(p+q)} \\
& \times\left[\int_{x_{0}}^{x}\left[\int_{x_{0}}^{t} V(s)|(L y)(s)|^{p+q} d s\right]^{p / q} V(t)|(L y)(t)|^{p+q} d t\right]^{q /(p+q)} \\
= & C A(x) \int_{x_{0}}^{x} V(t)|(L y)(t)|^{p+q} d t .
\end{aligned}
$$

This is the required inequality in (5) and the proof is complete.

The counterpart of the Theorem 1 is embodied in the following theorem.

Theorem 2. Let $x \leq x_{0}, x_{0}, x \in I$ and $p \geq 1, q>0$ be real constants. Let $U, V, \phi_{1}, \ldots, \phi_{n}, y$ be as in Theorem 1 . Then

$$
\int_{x}^{x_{0}} U(t)|y(t)|^{p}|(L y)(t)|^{q} d t \leq C B(x) \int_{x}^{x_{0}} V(t)|(L y)(t)|^{p+q} d t,
$$

where $C$ is defined as in Theorem 1 and

$$
\begin{aligned}
B(x)= & {\left[\int _ { x } ^ { x _ { 0 } } U ^ { ( p + q ) / p } ( t ) V ^ { - q / p } ( t ) \left[\int_{t}^{x_{0}} V^{-1 /(p+q-1)}(s)\right.\right.} \\
& \left.\left.\times\left(\sum_{k=1}^{n}\left|\phi_{k}(t) \sigma_{k}(s)\right|\right)^{(p+q) /(p+q-1)} d s\right]^{p+q-1} d t\right]^{p /(p+q)},
\end{aligned}
$$

for $x \leq x_{0}, x, x_{0} \in I$.

Proof. Let $x \leq t \leq x_{0}, x_{0}, x \in I$. From the hypotheses and using the Hölder's inequality with indices $p+q$ and $(p+q) /(p+q-1)$ we obtain

$$
|y(t)|^{p}=\left|\int_{t}^{x_{0}} \sum_{k=1}^{n} \phi_{k}(t) \sigma_{k}(s)(L y)(s) d s\right|^{p}
$$




$$
\begin{aligned}
\leq & {\left[\int_{t}^{x_{0}} \sum_{k=1}^{n}\left|\phi_{k}(t) \sigma_{k}(s)\right||(L y)(s)| d s\right]^{p} } \\
= & {\left[\int_{t}^{x_{0}} V^{-1 /(p+q)}(s)\left(\sum_{k=1}^{n}\left|\phi_{k}(t) \sigma_{k}(s)\right|\right) V^{1 /(p+q)}(s)|(L y)(s)| d s\right]^{p} } \\
\leq & {\left[\left[\int_{t}^{x_{0}} V^{-1 /(p+q-1)}(s)\left(\sum_{k=1}^{n}\left|\phi_{k}(t) \sigma_{k}(s)\right|\right)^{(p+q) /(p+q-1)} d s\right]^{(p+q-1) /(p+q)}\right.} \\
& \left.\times\left[\int_{t}^{x_{0}} V(s)|(L y)(s)|^{p+q} d s\right]^{1 /(p+q)}\right]^{p} .
\end{aligned}
$$

Multiplying both sides of $(12)$ by $U(t)|(L y)(t)|^{q}$, integrating the resulting inequality over $\left[x, x_{0}\right]$ and using the Hölder's inequality with indices $(p+q) / p$ and $(p+q) / q$ and following the proof of Theorem 1 with suitable changes we get the desired inequality in (10). The proof is complete.

We note that, in a recent paper [1] Anastassiou has obtained inequalities similar to that of given in Theorems 1 and 2 involving Green's function $H$ associated for the operator $L$ in place of the Wronskin $W$ involved in our results. We note that one can very easily extend the results given in [1] involving the Wronskin $W$ in place of the Green's function $H$ involved in the results given in [1]. Here we do not discuss the details.

We also note that the Opial's inequality (1) is a special case of our theorems when $n=1$.

\section{References}

[1] G. A. Anastassiou, Opial type inequalities for linear differential operators, Math. Inequalities and Appl. $\mathbb{1}$ (1998), 193-200.

[2] E. A. Coddington, An Introduction to Ordinary Differential Equations, Prentice-Hall, Inc., Englewood Cliffs, 1961.

[3]. D. S. Mitrinović, Analytic Inequalities, Springer-Verlag, Berlin, New York, 1970.

[4] Z. Opial, Sur une inégalité, Ann. Polon. Math. 8 (1960), 29-32.

[5] B. G. Pachpatte, On Opial-type integral inequalities, J. Math. Anal. Appl. 120 (1986), 547-556.

[6] B. G. Pachpatte, On inequalities of Opial type, Demonstratio Mathematica 25 (1992), 35-45.

[7] B. G. Pachpatte, A note on inequalities related to Opial's inequality, Indian J. Pure Appl. Math. 25 (1994), 1031-1037.

[8] B. G. Pachpatte, On Opial type inequalities involving higher order derivatives, J. Math. Anal. Appl. 190 (1995), 763-773.

Department of Mathematics, Marathwada University, Aurangabad 431 001, (Maharashtra) India. 